\title{
Real-time observation of vibrational revival in the fastest molecular system
}

\author{
A. Rudenko ${ }^{1}$, Th. Ergler ${ }^{1}$, B. Feuerstein ${ }^{1,2}$, K. Zrost $^{1,3}$, C.D. Schröter ${ }^{1}$, \\ R. Moshammer ${ }^{1}$, and J. Ullrich ${ }^{1}$ \\ ${ }^{1}$ Max-Planck-Institut für Kernphysik, Saupfercheckweg 1, 69117 Heidelberg, Germany \\ ${ }^{2}$ Physikalisches Institut, Universität Heidelberg, 69120 Heidelberg, Germany \\ ${ }^{3}$ Research School of Physical Sciences and Engineering, The Australian National University, \\ ACT 0200, Australia
}

\begin{abstract}
After preparing a coherent vibrational wave packet in the hydrogen molecular ion by ionizing neutral $\mathrm{H}_{2}$ molecules with a $6.5 \mathrm{fs}, 760 \mathrm{~nm}$ laser pulse at $3 \times 10^{14} \mathrm{~W} / \mathrm{cm}^{2}$, we map its spatiotemporal evolution by the fragmentation induced with a second 6.5 fs laser pulse of doubled intensity. In this proof-of-principles experiment we visualize the oscillations of this most fundamental molecular system, observe a dephasing of the vibrational wave packet and its subsequent revival. Whereas the experimental data exhibit an overall qualitative agreement with the results of a simple numerical simulation, noticeable discrepancy is found in the characteristic revival time. The most likely reasons for this disagreement originate from the simplifications used in the theoretical model, which assumes a Franck-Condon transition induced by the pump pulse with subsequent field-free propagation of the $\mathrm{H}_{2}{ }^{+}$vibrational wave packet, and neglects the influence of the rotational motion.
\end{abstract}




\section{Introduction}

Time-resolved studies of ultra-fast molecular dynamics simultaneously address the fundamental physical challenge of visualizing and understanding the time-dependent evolution of a quantum system [1] and the basic desire of chemists to trace or even control chemical reactions in real time [2]. One of the most successful techniques providing direct information in the time domain employs the so-called pump-probe scheme, where the first (pump) laser pulse initiates the molecular dynamics in a controlled way, i.e. launches a bound or continuum wave packet, while its time evolution is then probed with a second pulse arriving at variable delay times [3].

The probe stage usually exploits different laser-induced phenomena including the absorption and scattering of light, fluorescence or ionization. As a common characteristic for most of the probe schemes, the wave packet is sensitively monitored while it passes some welldefined positions in phase-space (that are related to time) like, for example, a certain energy difference between potential surfaces depending on a generalized molecular position coordinate, a vicinity of the inner or outer turning points etc. Thus, exclusively detecting the time-dependent variation of the wave packet probability distribution in a particular phase space window in most cases requires a complex inversion procedure to infer the shape of the molecular wave function from the experimental data.

A more direct method enabling in principle the observation of a wave packet at any time or position in configuration space is the so-called Coulomb explosion imaging (CEI). This technique was originally developed for collisions of fast molecular beams with thin foils, where the cleanest application of CEI has been demonstrated [4,5]. Here, two or more electrons are "instantaneously" (t $\sim 0.1-1 \mathrm{fs}$ ) ionized or 'stripped', causing the explosion of the molecule due to the Coulomb repulsion of its ionic constituents. The time-scale of this 'stripping' has to be short enough to suppress possible structural changes during the ionization process. Under the further requirement that the momentum transfer to the nuclei during ionization is small with 
respect to the Coulomb explosion energies, subsequent measurement of (angle-resolved) kinetic energy spectra of the ionic fragments provides information about the molecular structure at the moment of explosion. If, in addition, the ionization probability is independent of the generalized molecular coordinate, for instance the relative positions of the vibrating, rotating or dissociating nuclei, the complete probability density, i.e. the square of the nuclear wave function is mapped "one-to-one" into the continuum (taking into account trivial phase space factors) and its reconstruction is straight forward.

Whereas all the above requirements are well fulfilled for a large subset of ionizing collisions with the foils making this kind of experiments very successful (see., e.g. [4,5]), this approach is essentially restricted to time-independent studies of the molecular structure (except of following the slow vibrational cooling of molecules in storage rings [6]). In order to extend its applicability to time-resolved investigations on the femtosecond time scale, a simplified version of this technique was later combined with the laser-based pump-probe scheme $[7,8]$. Whereas laser-induced Coulomb explosion imaging (LCEI) was found to be less precise in measuring stationary state molecular structures than collision-based CEI techniques, since even for the shortest state-of-the-art laser pulses the characteristic ionization times remain considerably slower than in collisions [9], the principle possibility to perform time-resolved measurements with superior femtosecond resolution makes a combination of LCEI approach with a pumpprobe scheme extremely attractive for ultra-fast dynamics imaging [10,11]. However, due to the inherent complexity of the probe-step, i.e. of (multiple) ionization of molecules in strong laser fields where, in addition, one has to integrate over the space- and time-dependent intensity distribution of the laser pulse, simulations of the outcome of such pump-probe experiments in most of the practical cases represent a severe theoretical challenge, especially if large molecules containing many electrons are used, and the interpretation of the data is often difficult preventing any direct comparison between theory and experiment. 
As a result, there is strong interest in experiments using the simplest molecular systems, such as the hydrogen or deuterium molecules and their molecular ions (see [12,13] and references therein) which do, however, represent a major challenge for the experiment due to the very short time scales involved of typically less than $20 \mathrm{fs}$. Beyond the desire to understand the salient features of time-dependent Coulomb explosion imaging, necessary to efficiently trace ultra-fast molecular dynamics in the future, the $\mathrm{H}_{2}{ }^{+}\left(\mathrm{D}_{2}{ }^{+}\right)$molecular ion is of fundamental importance and, as outlined below, represents an ideal laboratory system to investigate the nonlinear interaction of strong laser fields with molecules in general.

In this paper we present a prove-of-principles experiment on LCEI of an $\mathrm{H}_{2}$ molecule using a combination of a "Reaction Microscope" to map the full final-state momentum space of exploding ions and emitted electrons with an ultra-fast, $6.5 \mathrm{fs}$, pump-probe arrangement. Achieving a 300 as stability for delay-times as long as $400 \mathrm{fs}$ we trace dissociating as well as vibrating wave packets in $\mathrm{H}_{2}{ }^{+}$in real time. It is highlighted that a refined analysis of the revival structure observed in $\mathrm{H}_{2}{ }^{+}$wave packet time evolution will provide unique information on the details of the vibrational wave packet formed, i.e. on the various vibrational modes contained, as well as on the tunneling-ionization probe step. A profound understanding of the latter is, as indicated above, of decisive importance for future applications of the LCEI technology in the general context of larger and more complex molecules. Though similar studies could have been more easily performed with a $\mathrm{D}_{2}$ target, where the nuclear motion is considerably slower, we have deliberately chosen $\mathrm{H}_{2}$, since the revival structure sensitively depends on the subtleties of the vibrational population distributions as well as on the anharmonicity of the potential, and, thus, hydrogen data will be extremely valuable for the further analysis and comparison with the heavier isotope. As a side aspect, we have addressed the technical challenge of mapping the fastest existing molecular nuclear wave packets.

The paper is structured as follows: In Section 2, important specific properties of the $\mathrm{H}_{2}{ }^{+}$ molecular ion are described in some detail and the status of present experimental investigations 
is briefly reviewed. The description of the experimental set-up along with the explanation of single pulse data and a brief discussion of the choice of experimental settings are given in Section 3. In Section 4, the results of the pump-probe experiments are presented and discussed in comparison with simple numerical simulations. Conclusions and outlook are given in Section 5 . Atomic units (a. u.) are used throughout if not stated otherwise.

\section{The $\mathrm{H}_{2} / \mathrm{H}_{2}{ }^{+}$Molecule and Experimental State-of-the-Art}

As indicated before, some specific properties do make $\mathrm{H}_{2}^{+}\left(\mathrm{D}_{2}^{+}\right)$an ideal model laboratory system for exploring laser-molecule interactions apart from the fact that only two nuclei and one electron are involved in the dynamics. First, there exists only one bound potential curve, i.e. the electronic ground state, whereas all other electronically excited states are purely repulsive and converge towards the Coulomb $1 / R$ curve, where $R$ is the internuclear distance (see Fig. 1). Second, the degeneracy of the two lowest electronic states $1 \mathrm{~s} \sigma_{\mathrm{g}}$ and $2 \mathrm{p} \sigma_{\mathrm{u}}$ at large internuclear distances leads to a strong dipole interaction of these levels in the presence of external electrical fields, which is essential for the coupling of the system to laser radiation. Third, there is quite large difference in the ionization energies of $\mathrm{H}_{2}(15.2 \mathrm{eV})$ and $\mathrm{H}_{2}{ }^{+}(29.9 \mathrm{eV})$ strongly supporting a two-step treatment, where, starting with the neutral $\mathrm{H}_{2}$, the formation or fragmentation of $\mathrm{H}_{2}{ }^{+}$, both occurring within the same pulse, can be easily separated.

Moreover, the latter feature provides a natural and ideal basis for a pump-probe experiment. As illustrated in Fig. 1, the neutral molecule is readily ionized by a first (pump) laser pulse, creating the wave packet in the ionic state, which can either dissociate along the fielddistorted $2 \mathrm{p} \sigma_{\mathrm{u}}$ potential curve, or remain bound in the $1 \mathrm{~s} \sigma_{\mathrm{g}}$ potential, modified during the pump pulse and unperturbed afterwards. Removing the second electron by a probe pulse, the wave packet is projected onto the $\mathrm{H}^{+}+\mathrm{H}^{+}$repulsive Coulomb curve, and the internuclear distance $\mathrm{R}$ at the moment of explosion can be directly reconstructed using the Coulomb's law. 
Despite its fundamental importance, appealing properties and numerous theoretical investigations being at hand, only two pump-probe studies on $\mathrm{H}_{2}$ [14] and $\mathrm{D}_{2}$ [15] using 50 and 80 fs pulses, respectively, have been reported until the last year due to the extraordinarily severe requirements posed onto the laser pulse duration. Thus, for example, the vibrational motion in $\mathrm{H}_{2}{ }^{+}$occurs on a time scale of about $10 \mathrm{fs}$ that is even nowadays not reachable with commercial laser systems. Using longer pulses, an alternative approach based on the so called 'molecular clock' concept has been suggested [16-19] delivering time-resolved information as well. Here, the removal of one electron by the laser pulse starts the clock, launching both a continuum electronic and a bound nuclear wave packet, respectively. Part of the electronic wave packet, which, as a whole, is driven back and forth in the oscillating field, revisits the parent molecular ion at well defined time intervals, representing the "ticking" of the clock and, at the same time, an inherent ultra-fast "probe": During "recollisions" the $\mathrm{H}_{2}{ }^{+}$ion might be further ionized, the molecule Coulomb explodes, and the clock, i.e. the propagation of the bound-state $\mathrm{H}_{2}{ }^{+}$nuclear wave-packet, is stopped. Though providing superior sub-fs time-resolution since the return time is defined on this level of accuracy, this method suffers from the difficulties in the separation of the recollision Coulomb explosion events from the field-induced ones. Even more critically, it is intrinsically restricted to fixed return times of the recolliding electron (for a given laser frequency) and, by definition, cannot be used to study long-term field-free propagation of the wave packet.

Therefore, considerable efforts are presently being undertaken to realize "classical" pump-probe experiments with sub-10 fs time resolution. Very recently, time-resolved imaging of the simplest molecular reaction, the dissociation of the hydrogen [20-22] or deuterium [21-24] molecular ion has been reported, and the possibility to control its outcome [24] or manipulate the product energy spectra [20] has been demonstrated. Moreover, first signatures of the vibrational motion in bound $\mathrm{H}_{2}{ }^{+}$[21,22] and $\mathrm{D}_{2}{ }^{+}$[22-24] were observed within the last year. Here, the motion of the wave packet could be traced as long as $100 \mathrm{fs}$ and good qualitative agreement with 
the theoretical simulations of its time evolution [22-25] was found for $\mathrm{D}_{2}{ }^{+}$. Fast degeneration of the oscillatory motion was attributed to the dephasing between the eigenstates of the vibrational wave packet $[21,23,24]$ which are, however, expected to revive at later times $[24,25]$.

The phenomenon of quantum wave packet revival, first found in numerical studies of Rydberg atoms [26], has now been observed in different atomic and molecular systems, both experimentally as well as theoretically (for reviews see [27-29]). It has been increasingly used in the development of modern quantum control experiments to assist in the shaping of specific quantum states [30,31]. Studies of the vibrational revival structure of molecules with different isotopic composition have been shown to allow for isotope separation [32,33] yielding information on the vibrational eigenfrequencies and anharmonicities of the potential as well as on the decoherence times [29]. Thus, the experimental observation of vibrational wave packet revivals in the simplest molecular systems, were a straight-forward semi-classical analysis can be complemented with more sophisticated numerical simulations and, in the future, might be even compared to envisioned ab-initio predictions, is of fundamental interest.

\section{Experiment}

A sketch of the experimental setup is shown in Fig. 2. Linearly polarized radiation from a Kerr-lens mode locked Ti:sapphire laser at $795 \mathrm{~nm}$ central wavelength with $25 \mathrm{fs}$ pulse width (FWHM) and $3 \mathrm{kHz}$ repetition rate was spectrally broadened in a neon-filled hollow fiber $(80 \mathrm{~cm}$ length, $200 \mu \mathrm{m}$ inner diameter) and then compressed to $6.5 \mathrm{fs}$ full width at half maximum (FWHM) by chirped mirrors and a prism-compressor sequence. The central wavelength of the output pulses was $760 \mathrm{~nm}$. The main pulse sits upon a background of $\sim 5 \%$ of the peak intensity lasting for $\sim 100 \mathrm{fs}$. Pulse to pulse energy fluctuations were monitored during the experiment and did not exceed 5\%. Absolute calibration of the peak laser intensity was performed using a "kink" in the measured photoelectron momentum distribution from single ionization of $\mathrm{Ne}$, which corresponds to the maximum drift momentum of $2 \sqrt{U_{p}}$ that electrons can gain from the laser 
field [34] ( $U_{P}$ : ponderomotive potential). The laser beam was fed through a Mach-Zehnder type interferometer with one arm variable in length, providing two pulses separated by a time delay which can be scanned from 0 to $3300 \mathrm{fs}$ with a resolution of better than $0.3 \mathrm{fs}$. By measuring the interferometric autocorrelation directly at the reaction volume using the ion rate as non-linear signal we verify in situ the length of the pulse and the absolute time zero of the delay determined by the symmetry axis of the autocorrelation pattern. The latter was not noticeably changed during $\sim 50$ hours data acquisition time, confirming the excellent long-term interferometric stability of our system.

Both laser beams were focused to a spot size of $\sim 5 \mu \mathrm{m}$ into the collimated supersonic molecular gas jet at the center of an ultra-high vacuum chamber $\left(2 \times 10^{-11}\right.$ mbar $)$. Charged reaction fragments were guided onto two position-sensitive channel plate detectors by weak electric $(2 \mathrm{~V} / \mathrm{cm})$ and magnetic $(7 \mathrm{G})$ fields applied along the laser polarization axis. From the respective time-of-flights and positions on the detectors the full momentum vectors of the coincident recoil ions and electrons were calculated. By inspecting momentum conservation for multi-photon single ionization along the jet propagation, its internal temperature was determined to be about $3 \mathrm{~K}$ such that $\mathrm{D}_{2}$ molecules are well prepared in their vibrational ground state before the interaction. A more detailed description of the spectrometer and the coincidence measurement scheme can be found in $[35,36]$.

The intensity of the pump pulse was $3 * 10^{14} \mathrm{~W} / \mathrm{cm}^{2}$. This value was chosen in order to provide rather high $\mathrm{H}_{2}{ }^{+}$yield, and, at the same time, to suppress further fragmentation of the molecular ion as much as possible. The latter process would reduce the effective density of the prepared ionic target, and produce a delay-independent background contribution, thus, spoiling the signal-to-noise ratio of the pump-probe measurement. A proton energy spectrum created by the pump pulse alone is presented in Fig. 3a. The pronounced peak close to $0.3 \mathrm{eV}$ energy with a long tail towards higher values originates from $\mathrm{H}_{2}{ }^{+}$dissociation, whereas the broad structure beyond $2 \mathrm{eV}$ is mostly due to Coulomb explosion. Note that the Coulomb explosion peak usually 
observed at around $3 \mathrm{eV}$ for $\mathrm{H}_{2}\left(\mathrm{D}_{2}\right)$ fragmentation in longer pulses of similar intensity [36-40] is strongly suppressed and shifted towards higher energies. The $3 \mathrm{eV}$ structure originates from enhanced ionization of $\mathrm{H}_{2}^{+}$in the laser field at rather large internuclear distances (4 - 6 a.u.) [4043] which, for the 6.5 fs pulses used in the present experiments are only reached by the main part of the wave packet when the intensity has already significantly dropped down [36,40].

The choice of the probe pulse intensity is more difficult. In [11] it was suggested to use $4 \times 10^{15} \mathrm{~W} / \mathrm{cm}^{2}$ pulses in order to project all the $\mathrm{H}_{2}{ }^{+}$ions onto the repulsive potential surface independent of the actual internuclear separation. This would, on the one hand, allow for a direct mapping of the nuclear wave function as discussed in the introduction; but, on the other hand, has two severe drawbacks. First, it leads to a considerable fraction of Coulomb explosion events produced within the probe pulse alone via sequential double ionization of neutral $\mathrm{H}_{2}$ molecules, since the initial $\mathrm{H}_{2}$ population is not fully depleted by the pump pulse [14,25]. Second, compared to pulses of lower intensities, it would reduce the time resolution of the experiment. As pointed out in [8], the ultimate time resolution of the pump-probe measurement is limited by the width of the effective ionization window. Thus, due to the strongly non-linear behavior of tunneling ionization, the resolution will increase with decreasing intensity for pulses with identical FWHM in time. Since the shortest pulse duration we can achieve with the present experimental setup ( $6.5 \mathrm{fs})$ is only slightly shorter than half of the $\mathrm{H}_{2}{ }^{+}$vibrational period we aim to resolve $\left(\mathrm{T}_{\text {vibr }} \sim 15\right.$ fs for the low-lying states), we were limited in the maximum intensity of the probe pulse. The crucial importance of the interrelation between the pulse duration and the time scale of the process of interest has been illustrated in [22]: Whereas it turned out to be feasible to trace the signatures of vibrational motion in $\mathrm{D}_{2}{ }^{+}$with $10 \mathrm{fs}$ pulses, the oscillations of the bound-state wave packet in $\mathrm{H}_{2}{ }^{+}$, which moves faster, can be barely resolved with 8 fs pulse duration.

After several tests we set the intensity of the probe pulse to $6^{*} 10^{14} \mathrm{~W} / \mathrm{cm}^{2}$ since for this value we observed the best contrast in the pump-probe signal. A proton energy spectrum obtained with the probe pulse alone is shown in Fig. 3b. Whereas the Coulomb explosion peak is 
more intense than for $3 * 10^{14} \mathrm{~W} / \mathrm{cm}^{2}$ (compare Fig. 3a and $3 \mathrm{~b}$ ), it is still considerably suppressed compared to the case of longer pulses of the same intensity [36-40].

\section{Results and discussion}

Fig. 4 displays the kinetic energy spectrum of the protons as a function of the time delay between the pump and the probe pulses. For delays below 100 fs the data manifest slight modulations with the laser period due to the interference between the $\sim 5 \%$ intensity pedestal with the main pulse.

Three major contributions, indicated by numbers in Fig. 4, can be distinguished in the spectrum. First, similar to the single pulse data, there is an intense band at $0-1 \mathrm{eV}$ kinetic energy, which originates from the dissociation of $\mathrm{H}_{2}{ }^{+}$. There are two possible pathways contributing to this band. In one case (pathway 1a) the ionization of the neutral molecule and the dissociation of the molecular ion occur within the same pulse, either the pump or the probe:

$$
\text { pump or probe: } \mathrm{H}_{2} \rightarrow \mathrm{H}^{+}+\mathrm{H}+\mathrm{e}^{-} \text {(pathway la). }
$$

Alternatively, the $\mathrm{H}_{2}{ }^{+}$ion, which has been created in the pump pulse and remained bound until the pulse has gone, can be dissociated by the probe pulse:

$$
\text { pump: } \mathrm{H}_{2} \rightarrow \mathrm{H}_{2}^{+}+\mathrm{e}^{-} \text {; probe: } \mathrm{H}_{2}^{+} \rightarrow \mathrm{H}^{+}+\mathrm{H}(\text { pathway } 1 \text { ) } \text {. }
$$

Second, one can see a broad structure at $2-4 \mathrm{eV}$ energy, with pronounced vertical or slightly tilted stripes in the delay region between 200 - 300 fs. This band is due to Coulomb explosion, where, similar to the previous case, both ionization steps can either occur within one pulse (most likely the probe one, see Fig. $3 a$ and $3 b)$ :

$$
\text { pump or probe: } \mathrm{H}_{2} \rightarrow \mathrm{H}^{+}+\mathrm{H}^{+}+2 \mathrm{e}^{-}(\text {pathway } 2 a) \text {; }
$$

or the first electron can be removed by the pump pulse, and the second one by the probe, respectively:

$$
\text { pump: } \left.\mathrm{H}_{2} \rightarrow \mathrm{H}_{2}^{+}+\mathrm{e}^{-} \text {; probe: } \mathrm{H}_{2}^{+} \rightarrow \mathrm{H}^{+}+\mathrm{H}^{+}+\mathrm{e}^{-} \text {(pathway } 2 b\right) \text {. }
$$


For very small delays this band is shifted towards larger proton energies due to the higher laser intensities achieved in case of constructive interference between both pulses [20,36-39].

Finally, a clear delay-dependent structure starting from the same energy region as the Coulomb explosion band discussed above close to zero time, and propagating towards lower and lower energies with increasing delay can be observed. At about 150 fs it merges with the dissociation band. This path reflects the process, where the $\mathrm{H}_{2}$ molecule undergoes dissociative ionization in the first pulse, and the dissociating $\mathrm{H}_{2}{ }^{+}$ion is ionized by the second one (the socalled ‘delayed ionization' [15,20]):

$$
\text { pump: } \mathrm{H}_{2} \rightarrow \mathrm{H}^{+}+\mathrm{H}+\mathrm{e}^{-} \text {; probe: } \mathrm{H}^{+}+\mathrm{H} \rightarrow \mathrm{H}^{+}+\mathrm{H}^{+}+\mathrm{e}^{-} \text {(pathway 3). }
$$

In [20-22] it was shown that the motion of the center of gravity of this dissociating wave packet can be reconstructed assuming its classical propagation with constant velocity. The measured kinetic energy release, i.e. the sum energy of both emitted protons then consists of the Coulomb energy and kinetic energy at the moment of explosion: $2 \mathrm{E}=1 / \mathrm{R}(\tau)+\mathrm{E}_{\mathrm{kin}}$, where the latter is given by the asymptotic value of the dissociation energy $\mathrm{E}_{\mathrm{diss}}$, which can be obtained from the single pulse measurements.

In the Coulomb explosion band (2) a series of clear stripes can be observed for delays between 200 - $300 \mathrm{fs}$, accompanied by maxima and minima in the dissociation band $(1+3)$. Similarly, inspecting in more detail the delay region $\tau<75$ fs (see the inset of Fig. 4), one finds a series of descending structures in the Coulomb explosion region $(\sim 2-4 \mathrm{eV})$ which degenerate into an unstructured band for larger delays between about $80-150 \mathrm{fs}$ as well as beyond $\sim 350 \mathrm{fs}$. These stripes (separated by $\sim 18 \mathrm{fs}$ ) originate from pathway $2 b$ described above and reflect the oscillatory motion of the $\mathrm{H}_{2}^{+}$vibrational wave packet created by the first pulse. As discussed in Section 2, a coherent superposition of several $\mathrm{H}_{2}{ }^{+}$vibrational states, prepared by the first pulse, dephases after a few oscillations due to the unequal spacing of the vibrational levels in the anharmonic molecular potential. However, after about 200 fs the wave packet partly regains its original form, exhibiting again an apparent periodicity of $\sim 18$ fs. As can be seen more clearly 
from Fig. 5, this revival structure appears in both, the Coulomb explosion and dissociation bands (in the latter case it is due to the pathway $1 \mathrm{~b}$ ). Thus, vibrational wave packets created by the pump pulse under current experimental conditions can be probed via both of these channels. However, as will be discussed below, rather different information might be extracted and different parts of the original wave packet, containing different vibrational mode composition are expected to dominantly contribute to the two pathways. It should be noted that the real contrast of the revival structure in the dissociation channel is considerably higher than the one that is visible in Figs. 4-5, because there the contribution from the pathway $1 b$ merges with that from the 'delayed ionization' of the ions dissociated in the first pulse (pathway 3), were no revival structure is expected.

Energy- and, thus, R-resolved imaging of the vibrational revival structure represents a major step forward in tracing and analyzing the wave packet dynamics as was shortly sketched in the introduction. Experiments detecting the ionization yield only (see, e.g. [44]) exploit and, more importantly, even rely on the fact that a particular ionization process favors the transition of the wave packet near a particular internuclear separation $\mathrm{R}$ (the so-called Franck-Condon point). In such experiments the signal is expected to "come and go" periodically as the wave packet travels through the specific detection window. The width of this window and thus, the characteristics of the detected signal, not only depends on the length of the probe pulse used but, moreover, is strongly affected by the details of the R-dependent process involved, i.e. for example, the R-dependent difference between the potential surfaces $\Delta \mathrm{E}(\mathrm{R})$ and on the energy uncertainty of the probe laser used, selecting this specific $\Delta \mathrm{E}(\mathrm{R})$, or on the sensitivity of a nonlinear process on a certain internuclear distance. More importantly, there might be situations where no $\Delta \mathrm{E}(\mathrm{R})$ fitting to available (one- or two-photon) laser frequencies does exist at all, as is often true for ground states of neutral molecules being strongly separated in energy from the first excited states, such that only few time-dependent studies of neutral molecule wave packet dynamics do exist at all. In contrast, in an energy-resolved CEI measurement the complete R- 
distribution of the wave packet at any probe time can, in principle, be directly observed provided the probe pulse is intense enough to ionize the molecule independently of R. In other words, the "effective R-window" can be chosen at will by selecting certain fragmentation energies. In this sense, the observation of the revival structure in the dissociation channel, which we probe at the same time, resembles a "conventional" experiment were the detection window is represented by all $\mathrm{R}$ where dissociation via Floquet-states occurs which is, most likely, close to the outer turning point.

In a LCEI experiment the accuracy of the R-window definition will depend on the probepulse temporal width, the fragment energy resolution of the spectrometer and, last but not least, on the width of the momentum distribution transferred to the nuclear fragments during the ionization process. Obviously, the scheme is applicable in any situation, where the probe pulse is strong enough to remove "some" electrons from the molecule causing Coulomb explosion. In the present case the ideal condition, namely the R-independence of the ionization probability, is not completely fulfilled. For a probe pulse intensity used in this work, the ionization probability is considerably increased at larger $\mathrm{R}$ leading to the weighting of the wave-packet images more strongly at smaller proton energies, i.e. at larger internuclear distances. In other words, the measured distributions at a given time occur shifted towards larger R. In the here described situation, the potential curves of $\mathrm{H}_{2}^{+}$are well-known, and, thus, it's R-dependent ionization probability $\mathrm{P}(\mathrm{R})$ can be calculated within a rather simple tunneling model [45], enabling a direct comparison between theory and experiment, which is illustrated in Fig. 6.

Fig. 6a displays the results of a simulation of the R-dependent vibrational wave packet dynamics of $\mathrm{H}_{2}{ }^{+}$assuming a vertical (Franck-Condon) transition from the ground state of $\mathrm{H}_{2}$. The data were obtained by solving numerically the one-dimensional time-dependent Schrödinger equation using the Crank-Nikolson split-operator method [46] (for details of the calculation see $[25,47,48])$. Fig. $6 \mathrm{~b}$ shows the results of an analogous simulation, where, however, the probability density was 'weighted' with the R-dependent ionization probability. The latter was 
calculated using the so-called "molecular ADK" formula [45] (ADK stays for Amosov, Delone, Krainov [49]). Finally, in Fig. 6c the time dependent R-distribution reconstructed from the experimental data of Fig. 4, is shown.

The comparison of Fig. $6 \mathrm{a}$ and $6 \mathrm{~b}$ illustrates how the R-dependence of the ionization probability affects the Coulomb explosion imaging of the vibrational wave packet. As a result of the R-dependent 'weighting', in Fig. $6 \mathrm{~b}$ the contributions from the inner region ( $\mathrm{R}<3$ a.u.) are strongly suppressed, and the rich structure in the outer region, including a broad band promptly ascending towards larger $\mathrm{R}$ within the first $50 \mathrm{fs}$, becomes more pronounced. A similar tendency can be traced in the experimental data of Fig. 6c. Both in the experimental data and in the calculation, two main contributions can be distinguished. The first one appears as an ascending band mentioned above reflecting the part of the wave packet that dissociates along the fieldmodified $2 \mathrm{p \sigma}_{\mathrm{u}}$ potential curve (giving rise to pathway 3 in the energy spectrum of Fig. 4 ). The second contribution is due to the wave packet bound in the $1 \mathrm{~s} \sigma_{\mathrm{g}}$ potential. While in Fig. 6a this part of the wave packet appears as a broad band between $\mathrm{R} \sim 1.5$ a.u. and $\mathrm{R} \sim 4$ a.u. (approximately corresponding to the inner and outer turning points, respectively), in Fig. $6 \mathrm{~b}$ it lays in the region of $\mathrm{R} \sim 3-7$ a.u..

A similar shift is apparent in the experimental data (Fig. 6c). However, there the corresponding band is clearly centred at $\mathrm{R} \sim 4$ a.u., whereas the structures extending beyond 5 a.u. are hardly resolved. The reason for this is a well known phenomenon, namely the so-called enhanced (or 'charge- resonance' enhanced) ionization mentioned in Section 3. This effect favors ionization at certain internuclear distances (4-5 a.u. for the case of $\mathrm{H}_{2}^{+}$) [41-43], in addition to the trivial $\mathrm{R}$ - dependence of the ionization potential, and is not taken into account in the simple tunneling model used in the calculation presented in Fig. 6b. Here it should also be noted that the internuclear distance distribution of Fig. 6c reconstructed from the measured energy spectra, was not corrected for the kinetic energy of the wave packet at the moment of explosion, since the latter is not precisely known, in contrast to the energy of the dissociating 
wave packets [20]. Taking the single pulse dissociation energy distribution peaked at $0.3 \mathrm{eV}$ (see Fig. 3), this correction can shift the measured R distribution up to 0.5 a.u. towards larger R.

Similar to the experimental data of Figs. 4-5, a fast dephasing after a few oscillations and a subsequent revival of the wave packet can be observed in all three panels of Fig 6. However, despite the qualitative similarity of the wave packet evolution predicted by the simulations and observed in the experiment, the revival structure in the calculation is noticeably shifted towards larger times. This can be more clearly seen in Fig. 7, were the measured Coulomb explosion and dissociating yields, as well as the calculated Coulomb explosion yield and mean value of $\mathrm{R}$ are shown as a function of the delay between the pump and the probe pulses. Comparing the calculated and measured time evolution of the proton yields from the Coulomb explosion channel (Fig. $7 \mathrm{~b}$ and $7 \mathrm{c}$, respectively), one finds that the structure of the revival as well as the time region, where the highest contrast of the ionization signal is achieved (the "revival time"), are considerably different.

The exact form and occurrence time of the revival is determined by the anharmonicity of the potential and by the distribution of the initial vibrational state population. According to equation (8) of [29], a localized wave packet with an energy spectrum tightly spread around a central quantum number $\mathrm{n}_{0}$ can be associated with the semiclassical "revival time" $\mathrm{T}_{\text {rev }}\left(\mathrm{n}_{0}\right)$, which is defined by the second-order term in the energy eigenvalues' expansion about $\mathrm{n}_{0}$. Using the tabulated energy values for the $\mathrm{H}_{2}{ }^{+}$molecular ion [50], we have calculated its semiclassical "revival times" varying the value of $\mathrm{n}_{0}$ (in this case it corresponds to the vibrational quantum number v) through all bound vibrational levels of $\mathrm{H}_{2}{ }^{+}$. The resulting time region is marked with the shaded area in Fig. 7, with the left and right boundaries corresponding to $v_{0}=18$ and $v_{0}=0$, respectively. The semiclassical "revival time" decreases almost linearly with the increasing $v_{0}$. Note that in the terminology of [27,29] the revival observed in this work is basically a halfrevival, where the wave packet reforms itself close to its original shape with nearly classical periodicity, but at a half-period off phase with respect to the initial wave form. As it can be seen 
from Fig. 7, the revival structure predicted by the numerical simulations fits to the semiclassically defined times. Though the experimental data exhibit clear oscillations in this region, both, in the dissociation and Coulomb explosion channels, for the latter (Fig. 7c) the highest contrast of the signal is observed before this region.

There are three possible reasons which might be responsible for the discrepancy between the measured and calculated revival times. The first one is related to the limitations of the theoretical model used. Indeed, the initial distribution of the $\mathrm{H}_{2}{ }^{+}$vibrational population was obtained assuming a vertical (Franck-Condon) transition from the ground state of $\mathrm{H}_{2}$. However, the distribution observed in a recent experiment (though produced by a considerably longer pulse) was found to be quite different from the Franck-Condon one [51]. Another reason might originate from the possible distortion of the field-free $\mathrm{H}_{2}{ }^{+}$potential curves induced by the laser pulses. As it was mentioned in Section 2, both, the probe and the pump pulses sit on a pedestal of $\sim 5 \%$ of the peak intensities lasting for almost 100 fs. That might increase the anharmonicity of the system, which, in turn, would lead to a shorter revival time [29,52]. Thus, a rigorous theoretical description of the wave packet preparation (ionization of the neutral $\mathrm{H}_{2}$ by the pump pulse) accounting for the exact form of the laser electric field, is required in order to reproduce the real vibrational state distribution.

The last but not least issue that considerably influences the observed vibrational revival structure concerns the role of the rotational motion. In numerous pump-probe experiments with $\mathrm{H}_{2}$ and $\mathrm{D}_{2}$ we do observe signatures of the rotational excitations induced by the 6-7 fs pump pulse in both, the neutral molecule or the molecular ion [53]. The ro-vibrational coupling, which was recently reported to play an important role in $\mathrm{H}_{2}$ revival dynamics [54], was not taken into account in the calculation and might contribute into the observed discrepancy between model calculation and experiment. However, the rotational motion modifies the observed vibrational revival structure also in a more direct way. In the calculations presented in Figs. 6-7 the molecular ion was assumed to be aligned along the laser polarization axes, whereas in the 
experiment the molecules are initially arbitrary oriented, and their orientation exhibits a rather complicated time-evolution after the pump pulse (see, e.g. $[55,56])$. The detected proton yields presented in Fig. $7 \mathrm{c}$ and $7 \mathrm{~d}$ depend on the orientation of the $\mathrm{H}_{2}{ }^{+}$ion at the moment when the probe pulse arrives because both, $\mathrm{H}_{2}{ }^{+}$ionization and dissociation probabilities in the linearly polarized laser field increase significantly for the molecules aligned parallel to the field polarization direction. On the other hand, the probability to doubly ionize, or to ionize and dissociate a neutral $\mathrm{H}_{2}$ molecule by the probe pulse alone providing a delay-independent background contribution, and, thus, changing the contrast of the pump-probe signal, depends on the orientation, and, consequently, on the rotational excitation of the neutral $\mathrm{H}_{2}$. The different role of the rotational motion of the molecular ion and of the neutral molecule might, along with the differences in the distribution of the contributing vibrational states discussed above, be the reason for the differences in the revival structure observed in the Coulomb explosion and dissociation channels in Fig. 7. Whereas the analysis of the time evolution of the rotational wave packets is not straight forward and is definitely beyond the scope of this paper, it seems to be essential for the rigorous theoretical description of the $\mathrm{H}_{2}{ }^{+}$vibrational revival.

\section{Conclusions and Outlook}

In this paper we have reported on the direct real-time mapping of a large part of $\mathrm{H}_{2}{ }^{+}$ vibrational wave packets created by coherent ionization of neutral $\mathrm{H}_{2}$ molecules with an intense $\left(3 * 10^{14} \mathrm{~W} / \mathrm{cm}^{2}\right)$, ultra-short $(6.5 \mathrm{fs})$ laser pulse. The time evolution of the wave packet is probed by another 6.5 fs pulse of doubled intensity via Coulomb explosion imaging and, simultaneously, via probe-pulse induced dissociation. Using a "Reaction Microscope" to analyze the kinetic energies and emission angles of all emitted ions, we provide a detection technology that is able to image the wave packet at any internuclear distance R. In this proof-of-principles experiment we visualize the vibrational motion in the fastest molecular system. 
In qualitative agreement with the numerical simulations of the wave packet propagation, we found a fast dephasing of the vibrational wave packet and its subsequent revival. Quantitatively, we observe noticeable discrepancy in the revival time predicted by our simple numerical model or by semiclassical analysis as compared to the experimental observation. The most likely reasons for this are the deviation of the pump-pulse produced $\mathrm{H}_{2}^{+}$vibrational distribution from a Franck-Condon one, assumed in our model, the distortion of the field-free $\mathrm{H}_{2}^{+}$potential by the long tail of the laser pulses used, and the influence of the rotational motion.

Further decrease of the probe pulse duration will allow one to use more intense pulses, thus, reducing the shift of the experimentally mapped probability distribution towards larger R. Moreover, extending the measuring time to delays as large as $3 \mathrm{ps}$, which is feasible with the present set up, the constituents of the wave packet can be determined explicitly with high accuracy by Fourier analysis of the time evolution of the revival structure. Then direct and very sensitive tests on strong-field $\mathrm{H}_{2}^{+}$vibrational wave-function formation should become feasible.

For diatomic molecules Coulomb explosion imaging used in the present work allows one to map not only the internuclear distance, but also the orientation of the molecule at the moment of explosion. Thus, exploiting angle-resolved data provided by our experimental set up, which we have recorded but not yet evaluated, it is possible to obtain snapshots of the complete nuclear wave function of the molecule at different times.

Finally we would like to stress, that also both electrons, the first one ejected during the pump step, and the second during the probe leading to Coulomb explosion, are mapped simultaneously - energy and angular resolved over the complete final state phase-space. In a test experiment, we were even able to "mark" the electrons by their different angular emission patterns using linear and circular laser polarization for the pump and probe pulses, respectively [53]. The analysis of the electron momentum distribution for the probe step then will provide unique information on the (small) momentum transfer during ionization $\vec{P}_{e}=-\left(\vec{P}_{H^{+}}+\vec{P}_{H^{+}}\right)$and, 
thus, enable to correct for it, providing ultimate precise Coulomb-explosion momenta for the heavy fragments.

Work is in progress into all above-mentioned directions. Moreover, future efforts will concentrate on the extension of our pump-probe imaging technique to other diatomics $\left(\mathrm{N}_{2}, \mathrm{O}_{2}\right.$, $\mathrm{CO}$ ), to more complicated molecules as well as to situations, where bonds are formed rather than broken during the laser pulse. In the more distant future, with the projected availability of intense attosecond pulses, one might envision to use a 300 as VUV pulse as a probe. It was predicted recently [57] that such a pulse will represent a "perfect" probe, ionizing the molecule with the same probability at any internuclear distance and, at the same time, with minimum momentum transfer (again detectable via electron momentum spectroscopy). Thus, the dream of following in real-time and with high-resolution ro-vibrational molecular motion and the possible coupling between the nuclear degrees of freedom as well as between the nuclei and the electrons, beyond the Born-Oppenheimer approximation, is deeming at the horizon.

\section{References}

[1] M.G. Raymer, Contemp. Phys. 38 (1997) 343.

[2] A.H. Zewail, J. Phys. Chem. 104 (2000) 5660.

[3] A. Mokhtari, P. Cong, J.L. Herek and A.H. Zewail, Nature 348 (1990) 225.

[4] Z. Vager, R. Naaman and E.P. Kanter, Science 244 (1989) 426.

[5] Z. Vager, T. Graber, E.P. Kanter and D. Zajfman, Phys. Rev. Lett. 703549 (1993).

[6] H. Kreckel, S. Krohn, L. Lammich, M. Lange, J. Levin, M. Sheffel, D. Schwalm, J.

Tennyson, Z. Vager, R. Wester, A. Wolf and D. Zaifman, Phys. Rev. A 66 (2002) 052509.

[7] H. Stapelfeldt, E. Constant and P.B. Corkum, Phys. Rev. Lett. 74 (1995) 3780.

[8] H. Stapelfeldt, E. Constant, H. Sakai and P.B. Corkum, Phys. Rev. A 58 (1998) 426.

[9] F. Légaré, K.F. Lee, I.V. Litvinyuk, P.W. Dooley, S.S. Wesolowski, P.R. Bunker, P. Dombi, F. Krausz, A.D. Bandrauk, D.M. Villeneuve and P.B. Corkum, Phys. Rev. A 71 (2005) 013415. 
[10] S. Chelkowski, P.B. Corkum and A.D. Bandrauk, Phys. Rev. Lett. 82 (1999) 3416.

[11] S. Chelkowski and A.D. Bandrauk, Phys. Rev. A 65 (2002) 023403.

[12] J.H. Posthumus, Rep. Prog. Phys. 67 (2004) 623.

[13] A. Giusti-Suzor, F.H. Mies, L.F. DiMauro, E. Charron and B. Yang, J. Phys. B 28 (1995) 309.

[14] J.H. Posthumus, J. Plumridge, P.F. Taday, J.H. Sanderson, A.J. Langley, K. Codling and W.A. Bryan, J. Phys. B 32 (1999) L93.

[15] C. Trump, H. Rottke and W. Sandner, Phys. Rev. A 59 (1999) 2858.

[16] H. Niikura, F. Légaré, R. Hasbani, A.D Bandrauk, M.Y Ivanov, D.M. Villeneuve and P.B. Corkum, Nature 417 (2002) 917.

[17] H. Niikura F. Légaré, R. Hasbani, M.Y Ivanov, D.M. Villeneuve and P.B. Corkum, Nature 421 (2003) 826.

[18] A.S. Alnaser, T. Osipov, E.P. Benis, A. Wech, B. Shan, C.L. Cocke, X.M. Tong and C.D. Lin, Phys. Rev. Lett. 91 (2003) 163002.

[19] A.S. Alnaser, X.M. Tong, T. Osipov, S. Voss, C.M. Maharjan, P. Ranitovic, B. Ullrich, B. Shan, Z. Chang, C.D. Lin and C.L. Cocke, Phys. Rev. Lett. 93 (2004) 183202.

[20] Th. Ergler A. Rudenko, B. Feuerstein, K. Zrost, C.D. Schröter, R. Moshammer and J. Ullrich, Phys. Rev. Lett. 95 (2005) 093001.

[21] Th. Ergler, A. Rudenko, B. Feuerstein, K. Zrost, C.D. Schröter, R. Moshammer and J. Ullrich, J. Phys B, accepted for publication.

[22] A.S. Alnaser, B. Ullrich, X.M. Tong, I.V. Litvinyuk, C.M. Maharjan, P. Ranitovic, T. Osipov, R. Ali, S. Ghimire, Z. Chang, C.D. Lin and C.L. Cocke, Phys. Rev. A 72 (2005) $030702(\mathrm{R})$.

[23] F. Légaré, K.F. Lee, I.V. Litvinyuk, P.W. Dooley, A.D. Bandrauk, D.M. Villeneuve and P.B. Corkum, Phys. Rev.A 72 (2005) 052717.

[24] H. Niikura, D.M. Villeneuve and P.B. Corkum, Phys. Rev. A 73 (2006) 021402 (R). 
[25] B. Feuerstein and U. Thumm, Phys Rev. A 67 (2003) 063408.

[26] J. Parker and C.R. Stroud Jr., Phys. Rev. Lett. 56 (1986) 716.

[27] I.Sh. Averbuch and N.F. Perelman, Sov. Phys. Usp., 34 (1991) 572.

[28] B.M. Garraway and A.-K. Suominen, Rep. Prog. Phys., 58 (1995) 395.

[29] R.W. Robinet, Phys. Rep., 392 (2004) 1.

[30] D.M. Meekhof, C. Monroe, B.E. King, W.M. Itano and D.E. Wineland, Phys. Rev. Lett. 76 (1996) 1796.

[31] D. Zeidler, A. Staudte, A.B. Bardon, R. Dörner, D.M. Villeneuve and P.B. Corkum, Phys. Rev. Lett. 95 (2005) 203003.

[32] I.Sh. Averbuch, M.J.J. Vrakking, D.M. Villeneuve and A. Stolow, Phys. Rev. Lett. 77 (1996) 3518.

[33] M. Leibsher and I.Sh. Averbuch, Phys. Rev. A 63 (2001) 043407.

[34] A. Rudenko K. Zrost, C.D. Schröter, V.L.B. de Jesus, B. Feuerstein, R. Moshammer and J. Ullrich, J. Phys. B 37 (2004) L407.

[35] V.L.B. de Jesus, A. Rudenko, B. Feuerstein, K. Zrost, C.D. Schröter, R. Moshammer, and J. Ullrich, J. Electron Spectrosc. Relat. Phenom. 141 (2004) 127.

[36] A. Rudenko, B. Feuerstein, K. Zrost, V.L.B. de Jesus, T. Ergler, C. Dimopoulou, C.D. Schröter, R. Moshammer and J. Ullrich, J. Phys. B 38, 487 (2005).

[37] T.D G Walsh, F.A. Ilkow and S.I. Chin, J. Phys. B 30 (1997) 2167.

[38] M.R. Thomson, M.K. Thomas, P.F. Taday, J.H. Posthumus, A.J. Langley, L.J. Frasinski and K.J. Codling, J. Phys. B 30 (1997) 5755.

[39] C. Trump, H. Rottke M. Wittmann, G. Korn, W. Sandner, M. Lein and V. Engel, Phys. Rev. A 62 (2000) 063402.

[40] F. Légaré, I.V. Litvinyuk, P.W. Dooley, F. Quéré, A.D. Bandrauk, D.M. Villeneuve and P.B. Corkum Phys. Rev. Lett. 91 (2003) 093002. 
[41] T. Zuo and A.D. Bandrauk, Phys. Rev. A 52 (1995) R2511.

[42] T. Seideman, M. Yu. Ivanov and P. B. Corkum, Phys. Rev. Lett. 75 (1995) 2819.

[43] M. Plummer and J.F. McCann, J. Phys. B 29 (1996) 4625.

[44] M.J.J. Vrakking, D.M. Villeneuve and A. Stolow, Phys. Rev. A 54 (1996) R37.

[45] A. Saenz, J. Phys. B 33 (2000) 4365.

[46] W.H. Press, S.A. Teukolsky, W.T. Vetterling, and B.F. Flannery, Numerical Recipes,

Cambridge University Press, Cambridge, 1992, p. 842.

[47] B. Feuerstein and U. Thumm, Phys. Rev. A 67 (2003) 043405.

[48] B. Feuerstein and U. Thumm, J. Phys. B 36 (2003) 707.

[49] M.V. Amosov, N.B. Delone and V.P. Krainov, Sov. Phys. JETP 64 (1986) 1191.

[50] G. Hunter, A.W. Yau and H.O. Pritchard, Atomic Data and Nuclear Data Tables 14 (1974) 11.

[51] X. Urbain, B. Fabre, E.M. Staicu-Casagrande, N. de Ruette, V.M. Andrianarijaona, J. Jureta, J.H. Posthumus, A. Saenz, E. Baldit and C. Cornaggia, Phys. Rev. Lett. 92 (2004) 163004.

[52] P. Domokos, T. Kiss, J. Janszky, A. Zucchetti, Z. Kis and W. Vogel, Chem. Phys. Lett. 322 (2000) 255.

[53] Th. Ergler, A. Rudenko, B. Feuerstein, K. Zrost, C.D. Schröter, R. Moshammer and J. Ullrich, to be published.

[54] J. Banerji and S. Ghosh, J. Phys. B, 39 (2006) 1113.

[55] I.V. Litvinyuk, K.F. Lee, P.W. Dooley, D.M. Rayner, D.M. Villeneuve and P.B. Corkum, Phys. Rev. Lett., 90 (2003) 233003.

[56] P. Dooley, I.V. Litvinyuk, K.F. Lee, D.M. Rayner, M. Spanner, D.M. Villeneuve and P.B. Corkum, Phys. Rev. A 68 (2003) 023406.

[57] C.D. Lin, X.M. Tong and T. Morishita, J. Phys B, accepted for publication. 


\section{Figure captions:}

Figure 1. Scheme of the pump-probe experiment and the relevant potential curves of $\mathrm{H}_{2}$ and $\mathrm{H}_{2}{ }^{+}$. The curves are shown for a field-free situation.

Figure 2. Sketch of the experimental set-up. BS: beam-splitter; PD: photodiode; MCP: microchannel plate.

Figure 3. Proton energy spectrum obtained by $\mathrm{H}_{2}$ fragmentation with the pump (a) and with the probe (b) pulse alone. Note that here, as well as in Figs. 4-5, the measured kinetic energy per proton is shown, and not the total kinetic energy release, as, for example, in [18,19,22].

Figure 4. A proton energy spectrum as a function of the delay between the pump and the probe pulses. Numbers in the figure indicate the pathways discussed in the text. Dashed oval indicates the revival region. Inset: zoom into the 'dephasing' region.

Figure 5. Same as Fig. 4 but for the revival region.

Figure 6. (a) Calculated density plot of $\mathrm{H}_{2}{ }^{+}$wave packet propagation (see text for details). (b) Same as (a) but 'weighted' with the R-dependent ionization probability (see text). (c) $\mathrm{H}_{2}{ }^{+}$wave packet reconstructed from the experimental data.

Figure 7. (a) Calculated mean value of the internuclear distance $\langle\mathrm{R}\rangle$; (b) calculated Coulomb explosion yield in the $3-4 \mathrm{eV}$ energy range; (c) measured Coulomb explosion yield in the $3-4$ eV energy range; (d) measured dissociation yield as a function of the delay between the pump and the probe pulses. Shaded area indicates the semiclassical revival times calculated for to $v_{0}=$ $0-18$ (see text). 


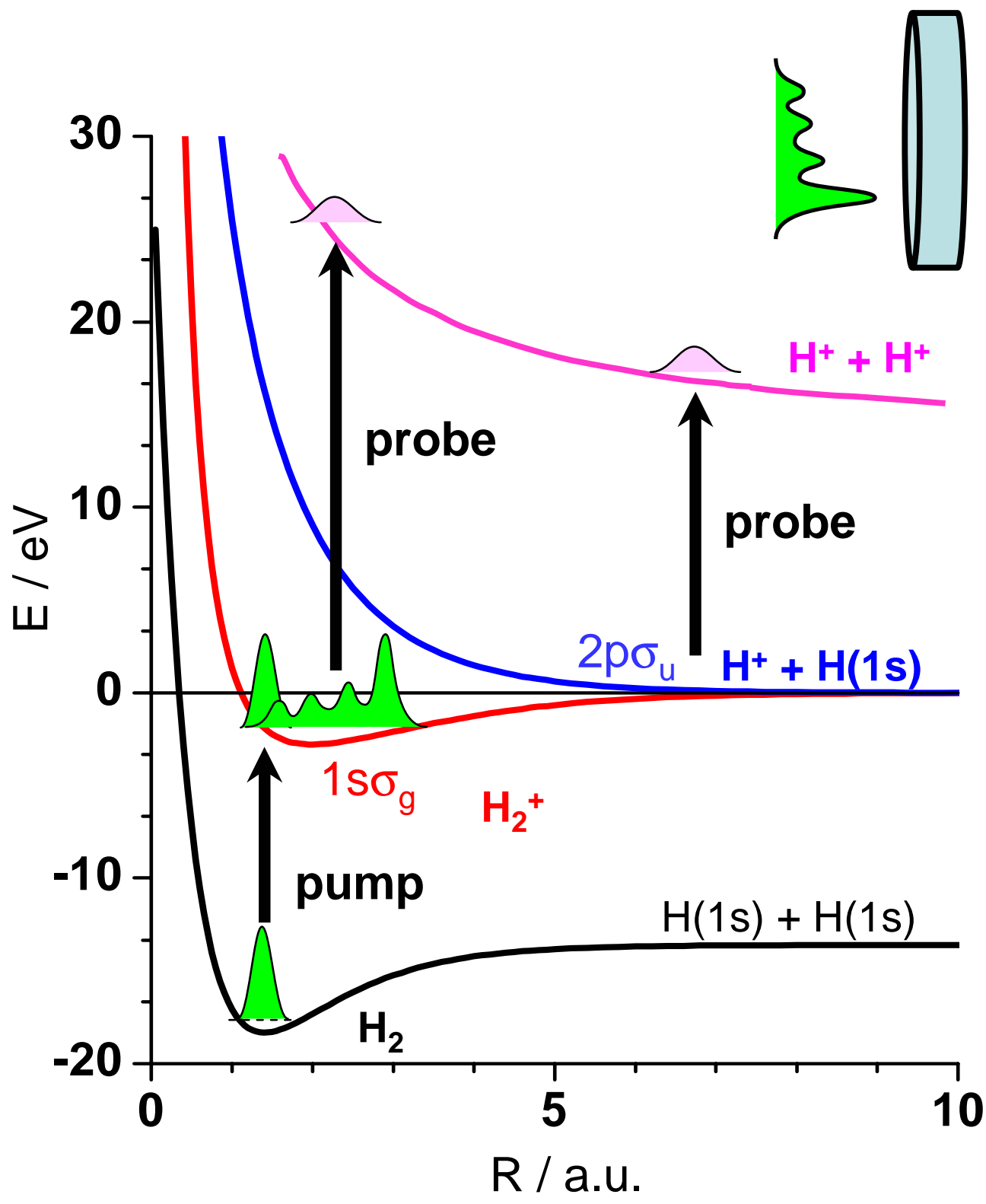

Figure 1 


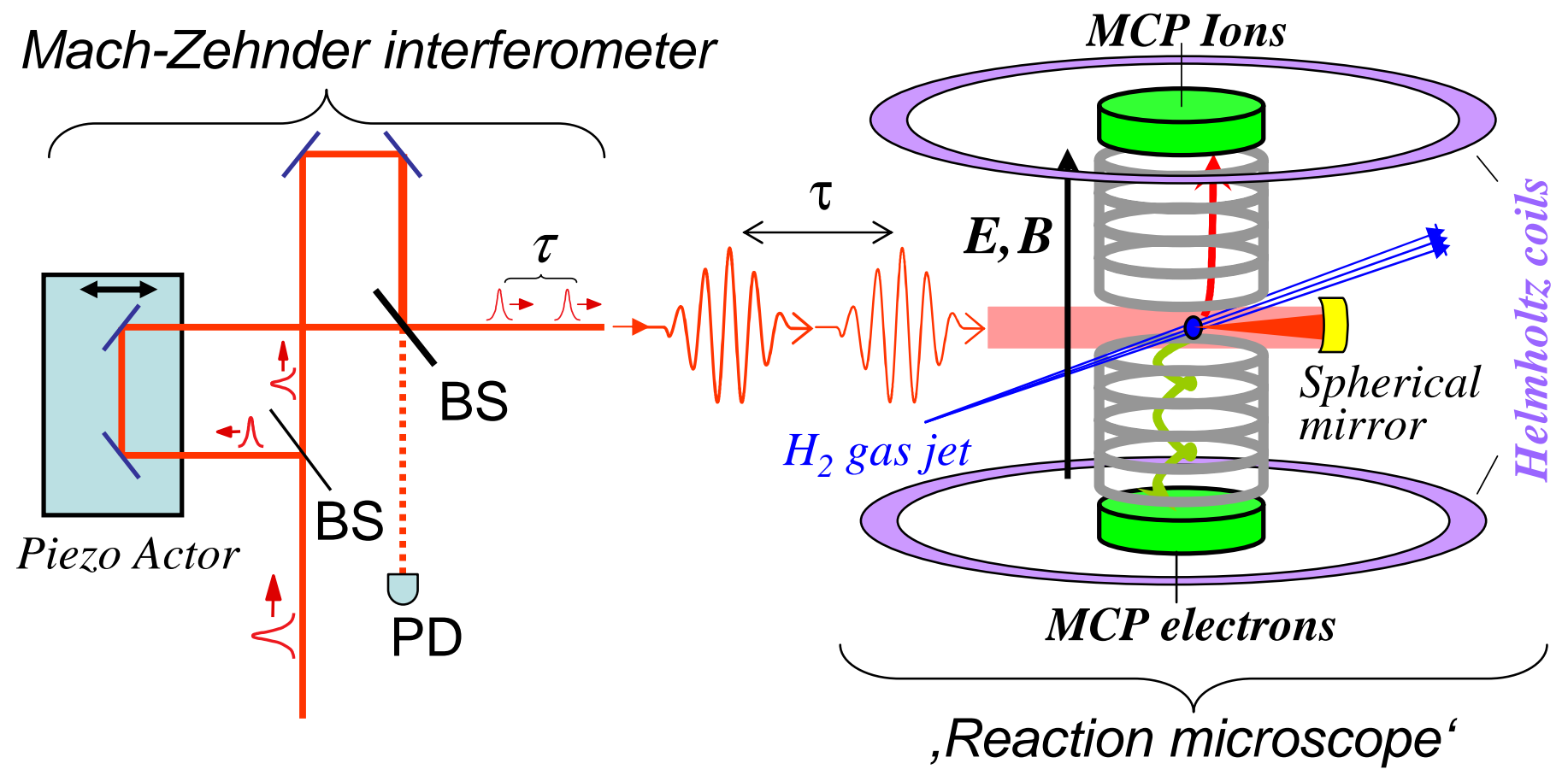

Figure 2 

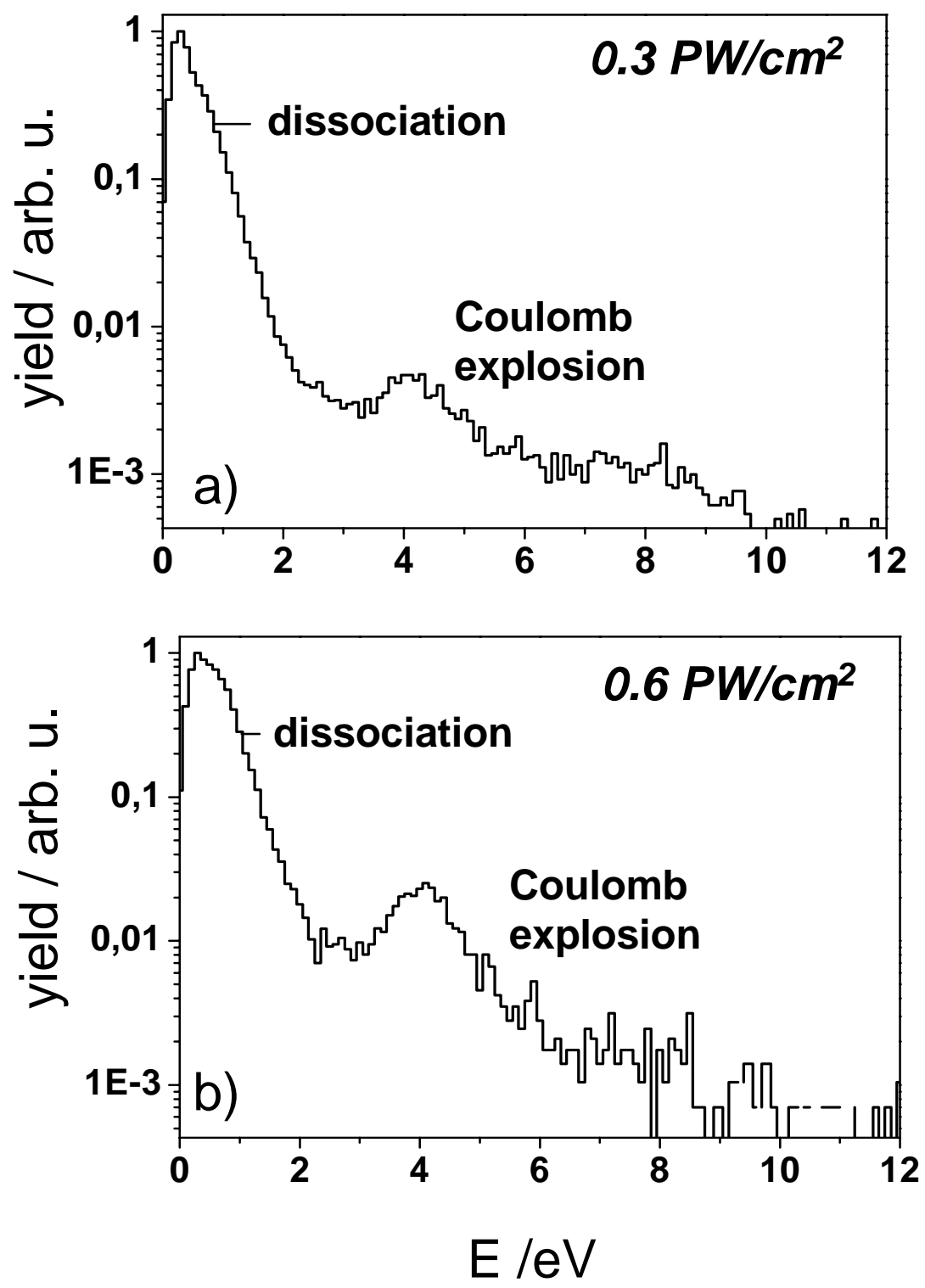

Figure 3 


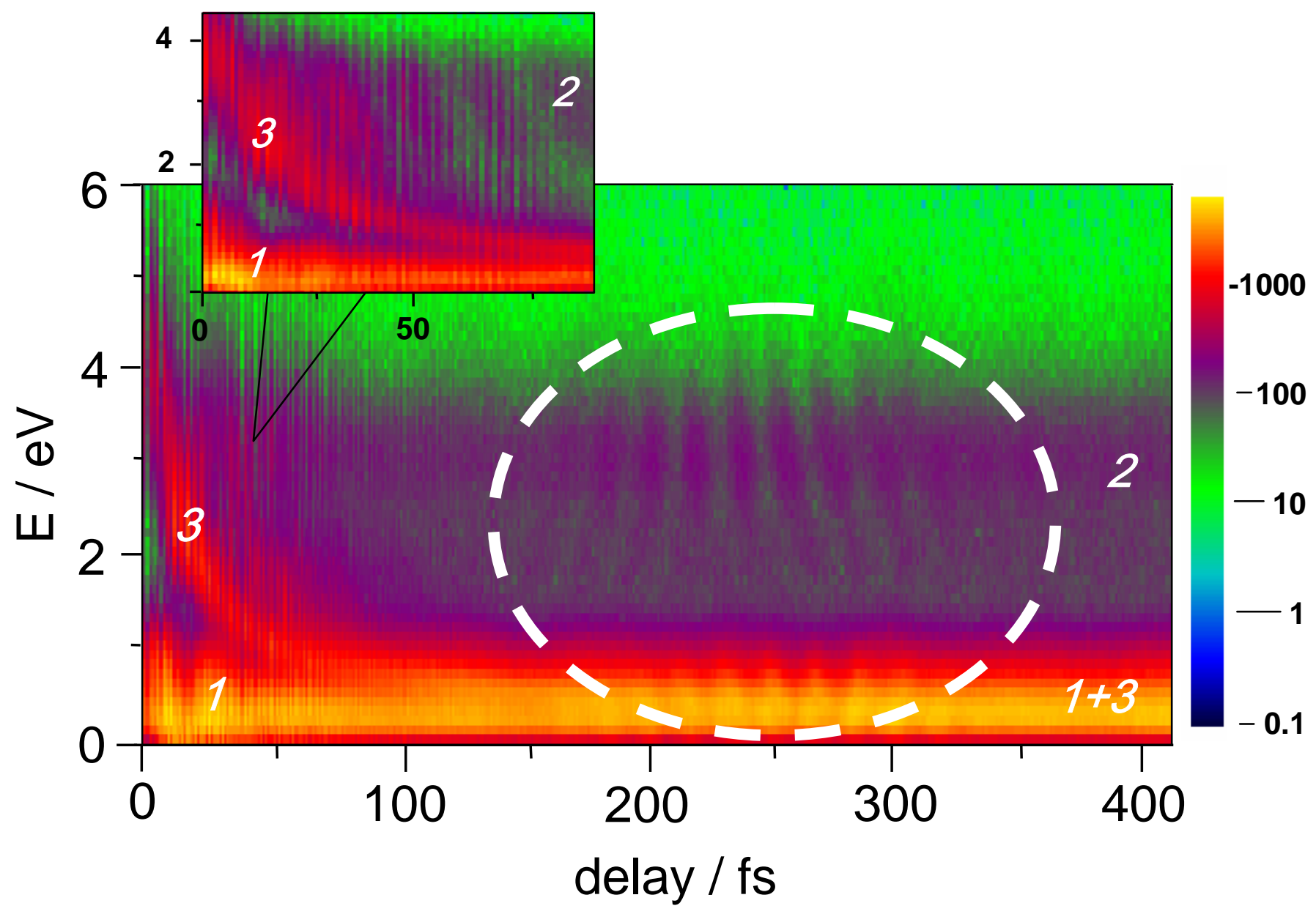

Figure 4 


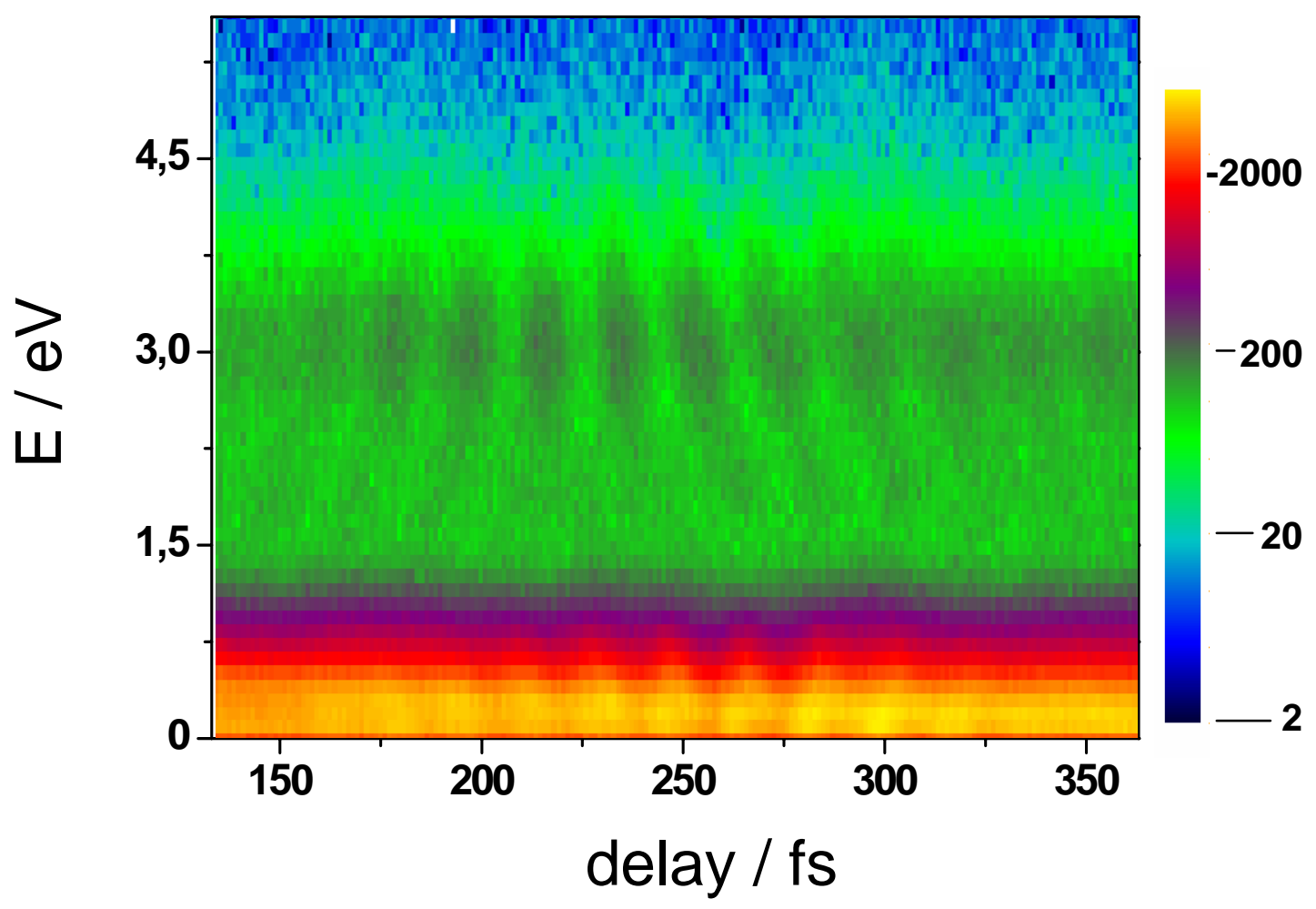

Figure 5 


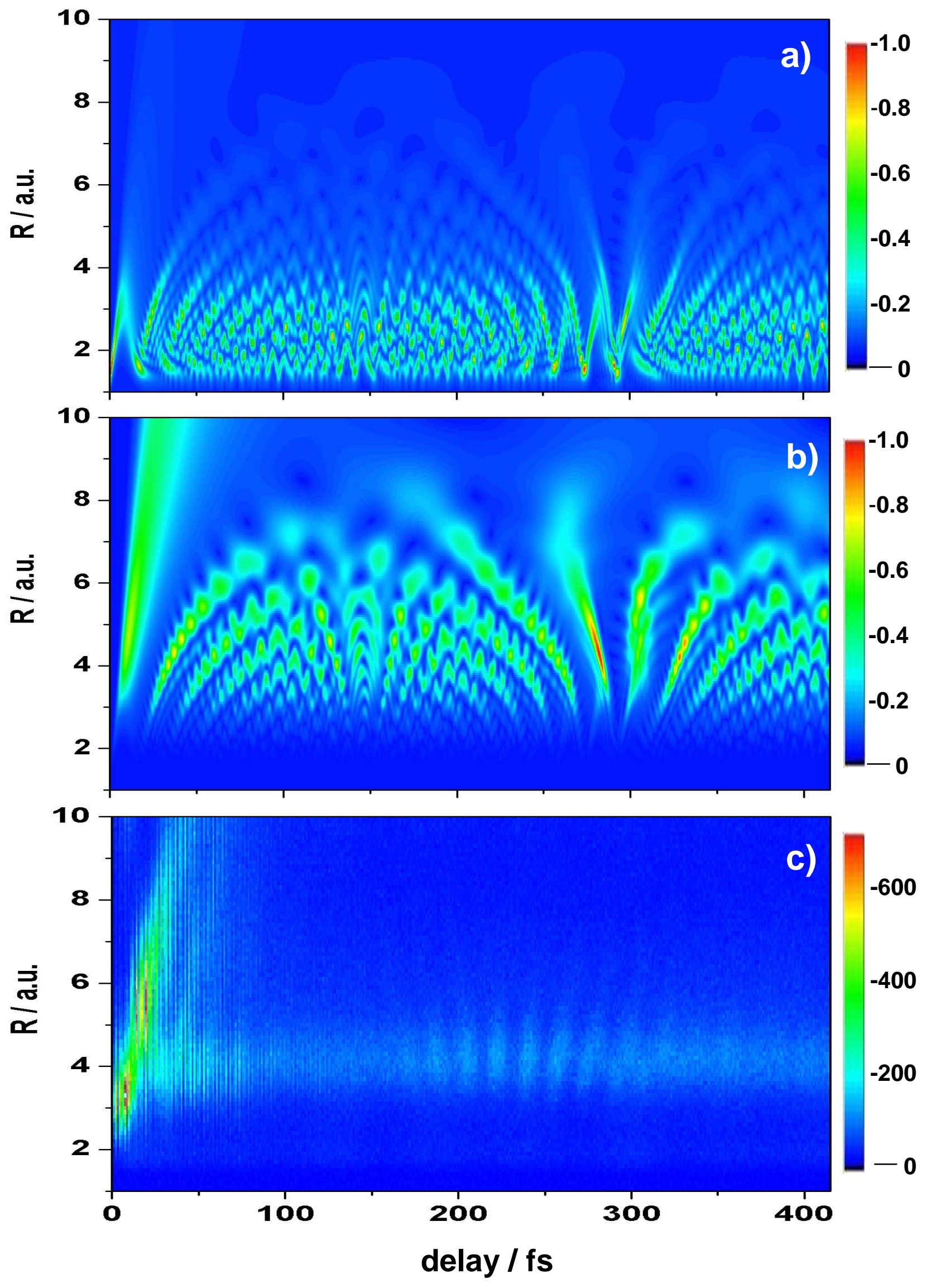

Figure 6 


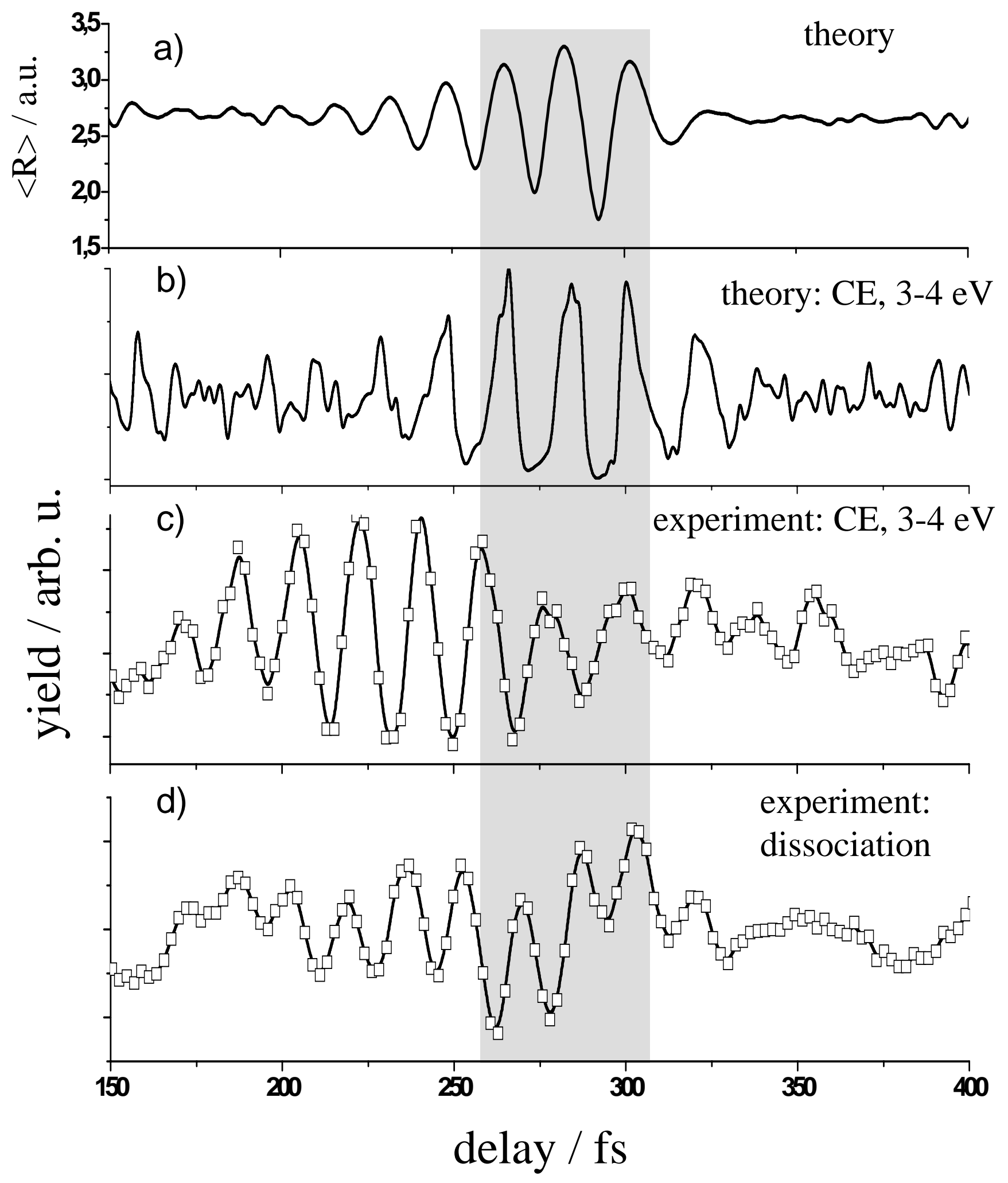

Figure 7 